\title{
Lost in translation-prognostic signatures for breast cancer
}

\author{
Lajos Pusztai
}

The number of prognostic gene signatures reported for breast cancer has increased dramatically in the past 5 years. The use of genomic prognostic assays, however, remains low, both in routine practice and in clinical trials. Currently, there are three commercially available genomic prognostic assays: Oncotype DX ${ }^{\circledR}$, MammaPrint ${ }^{\circledR}$, and $\mathrm{H} / \mathrm{I}^{\circledR}$. Only the Oncotype $D X^{\circledR}$ assay is used frequently in routine practice, although the other two assays were only recently introduced to the market. In addition, there are only two ongoing randomized clinical trials in breast cancer that use genomic assays for risk stratification: TAILORx and MINDACT. This situation might be considered reminiscent of the single-gene prognostic marker research field, where thousands of manuscripts were published over several decades but only three biomarkers, namely PR, ER, and HER2 were, and are still, used in the clinic routinely.

Nevertheless, genomic assays represent a clinically relevant and incremental improvement in molecular diagnostics. Prognostic signatures and classic clinical prognostic prediction models yield discordant results in about 25$30 \%$ of cases. ${ }^{1-3}$ In these cases, the genomic assays provide the more-accurate prognostic prediction. The improved prognostic information is most likely because of the more-accurate molecular risk stratification of the intermediate grade tumors and the more-reliable assessment of the proliferative activity of cancers using gene-expression signatures. ${ }^{4,5}$

Reporting significant associations between a molecular marker and an outcome does not make a test-it only makes a manuscript. A test requires definition of marker cut-off values (i.e. to identify when a sample is positive or negative), documentation of technical reproducibility, and validation of the cut-off values in independent cases. These data can be obtained through a series of prospectively designed clinical studies enrolling patients whose clinical characteristics match the intended use of the test. This systematic approach to biomarker development
Reporting

significant

associations

between a

molecular

marker and

an outcome

does not make

a test-it

only makes a

manuscript.

L Pusztai is Associate Professor of Medicine at The University of

Texas MD Anderson

Cancer Center,

Houston, TX, USA.

Competing interests

The author has declared an association with the

following company: Nuvera

Biosciences. See the article

online for full details of the

relationship.

www.nature.com/clinicalpractice doi:10.1038/ncponc1177 is advocated by many in the genomic research community. Clinicians have recognized for some time that a combination of clinical variables provide better prediction tools than single variables. High-throughput molecular assays have made it possible to apply this time-honored principle to molecular markers. It is customary to report progesterone receptor, ER, HER2, Ki67 and other markers individually; however, the best prognostic prediction comes from analyzing these markers in combination. The human mind is not apt to perform this calculation efficiently; therefore, combining individual variables into a single prediction score is another important conceptual step forward.

What contributes to the slow translation of promising signatures into clinically useful assays? The most important bottleneck might be the lack of sufficiently large numbers of samples from patients treated with different regimens that require validation. Many discovery and validation studies were performed on 'convenience samples' available through tissue banks. The clinical utility of the observations is limited by the varied treatments used, and the small sample sizes produce low confidence intervals in the accuracy of predictions. The validation of some assays is better than others but none is perfect. The challenge for investigators is to find the niche where the current imperfect tests provide clear clinical value and confront the limits of the current genomic predictors so that work on the next generation of improved assays can begin. Important challenges for the future include the development of prognostic markers for ER-negative and HER2-negative cancers, which are almost invariably regarded as high-risk by current genomic predictors, and to combine multiple separate predictive tests into a single assay to improve cost-effectiveness and increase information value.

Supplementary information in the form of a reference list is available on the Nature Clinical Practice Oncology website. 